08

\title{
Локализация носителей заряда в самоорганизованных квантовых точках InAs
}

\author{
() А.Н. Косарев ${ }^{1,2}$, В.В. Чалдышев ${ }^{1,9}$ \\ ${ }^{1}$ Физико-технический институт им. А.Ф. Иофрфе РАН, Санкт-Петербург, Россия \\ ${ }^{2}$ Санкт-Петербургский политехнический университет Петра Великого, Санкт-Петербург, Россия \\ E-mail: kosarev@mail.ioffe.ru \\ ฯ E-mail: chald.gvg@mail.ioffe.ru
}

Поступило в Редакцию 30 августа 2021 г.

В окончательной редакции 7 сентября 2021 г.

Принято к публикации 8 сентября 2021 г.

Рассмотрена проблема локализации электронов и дырок на примере пирамидальных квантовых точек InAs в арсениде галлия. Задача квантовой механики решена для локализующего потенциала с учетом геометрии, химического состава и встроенных полей механических напряжений и деформаций. Установлено, что наилучшая локализация носителей обоих типов достигается при отношении высоты пирамиды к ее основанию около 0.2 .

Ключевые слова: квантовые точки, упругие деформации, локализация носителей заряда.

DOI: 10.21883/PJTF.2021.23.51786.19006

Глубина и характер локализации электронов и дырок являются главными параметрами квантовых точек (КТ), определяющими их электронные и оптические свойства и возможности их использования в различных электронных, оптоэлектронных и фотонных устройствах $[1,2]$. Несмотря на значительную разницу ширин запрещенных зон материала, из которых формируется (самоорганизуются) КТ, и материала окружающего барьерного слоя, локализация носителей заряда в КТ часто оказывается слабой или вообще невозможной. Причиной этого являются механические деформации, наличие которых необходимо для процесса самоорганизации по механизму Странского-Крастанова [3,4]. Ярким примером этого явления служат KT InAs, технология получения (самоорганизации) которых в процессе молекулярно-лучевой эпитаксии хорошо разработана [5]. Поскольку ширина запрещенной зоны InAs составляет $0.4 \mathrm{eV}$, а ширина запрещенной зоны барьера $\mathrm{GaAs}$ составляет $1.5 \mathrm{eV}$, КТ InAs потенциально могли бы покрыть очень широкий и практически важный ближний инфракрасный оптический диапазон, включая окно прозрачности оптического волокна вблизи $1.55 \mu \mathrm{m}(0.8 \mathrm{eV})$, обычно используемое в линиях оптической связи. В реальности излучение из KT InAs имеет место в диапазоне длин волн $0.8-1.1 \mu \mathrm{m}$, возрастая с увеличением размеров КТ [6].

В настоящей работе рассмотрена проблема локализации электронов и дырок на примере пирамидальных КТ InAs в арсениде галлия. Мы установили, что при заданном объеме КТ глубина локализации носителей обоих типов имеет нетривиальную зависимость от отношения высоты пирамиды к ее основанию.

Мы провели расчеты для типичных КT InAs, получаемых методом молекулярно-лучевой эпитаксии на подложках (001) GaAs в режиме Странского-Крастанова при наращивании пяти монослоев InAs. Сформированные на поверхности роста пирамидальные КТ InAs заращивались барьерным слоем GaAs толщиной $30 \mathrm{~nm}$. Процедура выращивания и формирования системы КТ описана в работе [7]. Результаты детальных электронномикроскопических исследований таких КТ представлены в [8]. Эти исследования, в частности, показали, что типичная высота KT InAs, заращенных в GaAs, составляет $H=9.0 \pm 0.5 \mathrm{~nm}$, а основание пирамиды $L=28.0 \pm 0.5 \mathrm{~nm}$. При этом ребра основания пирамиды были параллельны [100] и [010]. Грани пирамиды соответствовали индексам Миллера \{203\}. Такие размеры и форма КТ были выбраны нами в качестве референтных. Следует отметить, что заращивание КТ приводит к уменьшению отношения высоты пирамиды к ее основанию. Этот эффект является типичным для КТ, сформированных путем самоорганизации на поверхности $[9,10]$. Проявление этого эффекта существенно зависит от условий заращивания. Кроме изменения соотношения размеров заращивание может также приводить к перемешиванию In-Ga. Для референтной KT мы приняли концентрацию индия однородной и равной 0.92 , что обеспечило согласование расчетов энергетических уровней с наблюдаемым энергетическим положением линии фотолюминесценции из КТ.

Использованный в расчетах подход описан в нашей работе [11]. Для определения глубины локализации электронов и дырок и последующего расчета спектрального положения пика экситонного излучения была численно решена задача квантовой механики для обоих типов носителей в однозонной модели. При этом локализующий потенциал был получен в результате решения задачи линейной теории упругости для заданной геометрии и однородного химического состава КТ. В процессе 
расчета варьировалось отношение высоты к основанию пирамиды $0.05<H / L<1.75$ при сохранении ее объема. Концентрация индия соответствовала референтной КТ и также сохранялась неизменной. Все расчеты проводились методом конечных элементов. Объем ячейки, в которой проводился расчет, в $\sim 450$ раз превосходил объем КТ. Параметры материала для расчетов были взяты из работ $[4,12]$. Определение параметров квазибинарного твердого раствора InAs-GaAs проводилось по правилу Вегарда. Ширины запрещенных зон для $\mathrm{GaAs}$ и InAs составляли 1.51 и $0.4 \mathrm{eV}$ соответственно для температуры $77 \mathrm{~K}$, при которой были выполнены экспериментальные исследования спектров экситонного излучения из референтной КТ.

Результаты расчета энергии упругой деформации для различных соотношений высоты и основания пирамиды $H / L$ приведены на рис. 1 . На рис. $1, a$ энергия проинтегрирована по всей ячейке, по которой проводился расчет, а на рис. 1, $b$ показана часть этой энергии, запасенная в объеме КT InAs. Видно, что уменьшение формфактора $H / L$ приводит к уменьшению полной энергии системы, что создает термодинамическую движущую силу, вызывающую уменьшение отношения $H / L$ при заращивании КТ. При этом часть полной упругой энергии, которая локализована внутри КТ, увеличивается.

Полная упругая энергия может быть представлена через компоненты тензоров механических напряжений $\sigma$ и упругих деформаций $\varepsilon$ :

$$
\begin{aligned}
W_{\text {total }} & =\int\left[\frac{1}{6} \operatorname{tr}(\varepsilon) \operatorname{tr}(\sigma)+\left(\frac{1}{2} \varepsilon_{i i} \sigma_{i i}-\frac{1}{6} \operatorname{tr}(\varepsilon) \operatorname{tr}(\sigma)\right)\right. \\
& \left.+\frac{1}{2} \varepsilon_{i j} \sigma_{i j}\right] d V .
\end{aligned}
$$

Здесь первый член в квадратных скобках представляет гидростатическую энергию. Второй член, который мы будем называть девиаторным, соответствует биаксиальной дисторсии. Последний член представляет энергию сдвиговых деформаций, описываемых недиагональными компонентами тензоров $\sigma$ и $\varepsilon$. Изменение этих вкладов в полную упругую энергию в зависимости от отношения $H / L$ представлено на рис. 1 . Из рисунка видно, что гидростатическая часть упругой энергии в основном аккумулирована внутри КТ, а упругая энергия вне КТ в основном обеспечивается девиаторными и сдвиговыми компонентами поля механических напряжений и деформаций. Этот результат хорошо согласуется с предсказаниями аналитических моделей включений [13-15].

В отличие от полной упругой энергии системы ее часть, аккумулированная внутри КТ, увеличивается при уменьшении отношения $H / L$. Важно, что это увеличение в основном обеспечивается девиаторной компонентой энергии. Объяснение этого факта состоит в том, что при уменьшении высоты и увеличении основания распределение механических напряжений и деформаций приближается к реализующемуся в тонкой пленке.

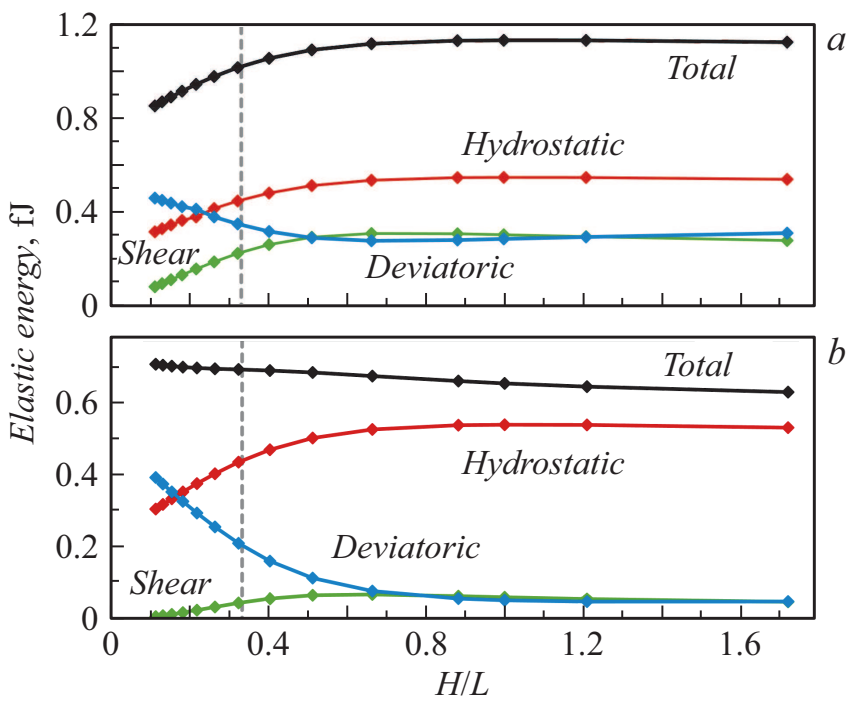

Рис. 1. Зависимость энергии упругой деформации от отношения высоты к основанию для пирамидальной КT InAs. Представлены кривые для полной энергии (Total), гидростатической компоненты (Hydrostatic), девиаторной компоненты (Deviatoric), сдвиговой компоненты (Shear). $a$ - энергия всей ячейки, $b-$ энергия только объема КТ.

Влияние механических полей на энергии электронов и дырок описывается через деформационные потенциалы и пьезоэлектрический эффект. Изменение потенциала электронов по отношению к недеформированному материалу пропорционально изменению объема элементарной ячейки кристаллической решетки:

$$
\delta E_{e}=a_{e} \operatorname{tr}(\varepsilon) .
$$

Здесь $a_{e}-$ отрицательный гидростатический деформационный потенциал электрона. Валентная зона InGaAs вырождена в центре зоны Бриллюэна, где находится ее потолок. Девиаторные и сдвиговые компоненты деформаций снимают это вырождение тяжелых и легких дырок в дополнение к гидростатическому сдвигу энергий

$$
\delta E_{h}=a_{h} \operatorname{tr}(\varepsilon) \pm \sqrt{\begin{array}{l}
\frac{b^{2}}{2}\left[\left(\varepsilon_{x x}-\varepsilon_{y y}\right)^{2}+\left(\varepsilon_{x x}-\varepsilon_{z z}\right)^{2}+\right. \\
\left.+\left(\varepsilon_{y y}-\varepsilon_{z z}\right)^{2}\right]+d^{2}\left(\varepsilon_{x y}^{2}+\varepsilon_{x z}^{2}+\varepsilon_{y z}^{2}\right)
\end{array}}
$$

Здесь $a_{h}-$ положительный гидростатический деформационный потенциал для дырок, $b$ и $d-$ деформационные потенциалы для девиаторной и сдвиговой компонент тензора упругих деформаций.

Наличие упругих деформаций вызывает в InGaAs пьезоэлектрический эффект. Наши расчеты показали, что вклад пьезоэлектрического эффекта в локализующий потенциал КТ относительно мал, и в большинстве случаев им можно пренебречь. К такому же выводу ранее пришли авторы работы [12], проводившие аналогичные расчеты для пирамидальных КT InAs с гранями $\{101\}$. 

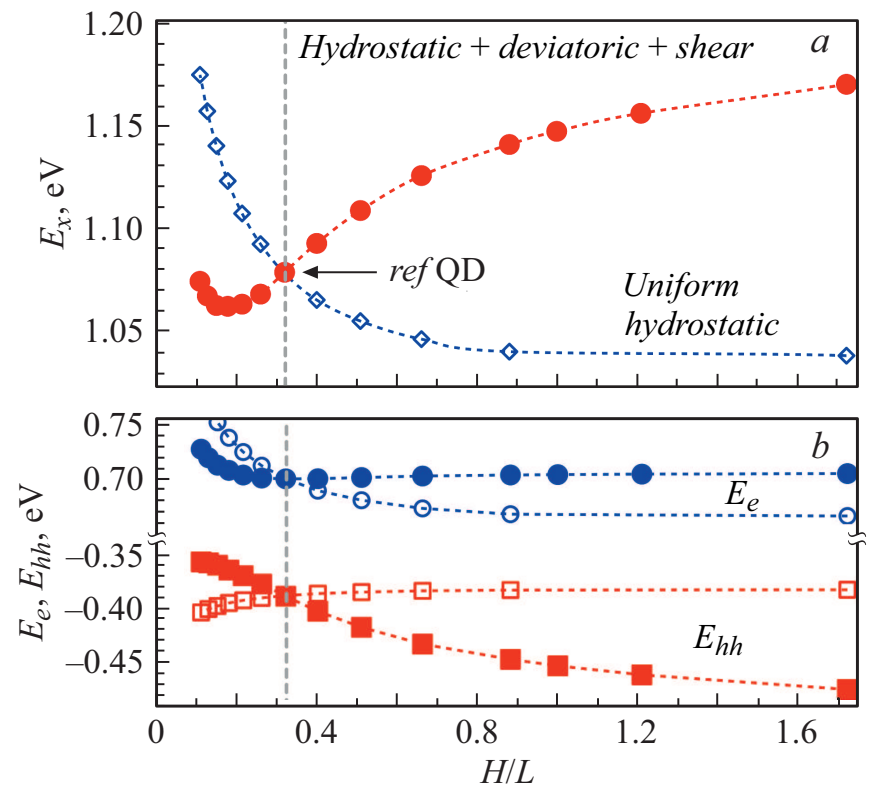

Рис. 2. Зависимости энергии экситона $(a)$, энергий электронов и тяжелых дырок $(b)$ от отношения высоты к основанию для пирамидальной КT InAs. Закрашенные символы - результат расчета с учетом всех компонент тензора деформаций, светлые - результат расчета с учетом только гидростатической части деформаций. Формфактор референтной КТ $H / L=0.32$ отмечен вертикальными штриховыми линиями и соответствует точкам пересечения кривых.

Результаты квантово-механического расчета энергий основного состояния электронов $E_{e}$ и дырок $E_{h h}$ в КТ InAs, окруженных барьером GaAs, в зависимости от отношения $H / L$ показаны на рис. 2, $b$ (закрашенные кружки и квадраты). Закрашенные кружки на рис. 2,a показывают соответствующую зависимость для энергии основного состояния экситона в КТ, вычисленную в предположении о независимости энергии связи экситона от отношения $H / L$. При изменении формфактора объем КТ сохранялся неизменным и равным объему референтной КТ. Формфактор референтной КТ $H / L=0.32$ отмечен на рис. 1 и 2 вертикальными штриховыми линиями. Полагая энергию связи экситона в КТ данного объема равной $10 \mathrm{meV}$ [12], мы получили количественное согласие результатов расчета с данными фотолюминесценции, полученными при $77 \mathrm{~K}$. Соответствующая точка отмечена на рис. 2, $a$ стрелкой.

Из рис. 2, $b$ видно, что энергия основного состояния электрона имеет минимум в окрестности $H / L=0.2$. Эта энергия быстро растет при малых $H / L$ вследствие усиления размерного квантования вдоль оси пирамиды. Относительно медленное возрастание энергии электронов при увеличении $H / L$ связано с увеличением гидростатической части деформаций, как видно из рис. 1.

Влияние размерного квантования на энергию основного состояния дырки в КТ выражено слабее из-за большей величины эффективной массы, чем в случае электрона.
При этом $E_{h h}$ смещается вверх по шкале энергий вследствие преобразования гидростатических деформаций в девиаторные при уменьшении отношения $H / L$.

В результате немонотонного изменения как энергии электронов, так и энергии дырок энергия экситонов, локализованных в КТ, имеет хорошо выраженный минимум, соответствующий отношению высоты и основания пирамиды $H / L=0.2$. По сравнению с пирамидальными КТ с неоптимальным формфактором выигрыш в энергии экситонов может превышать $0.1 \mathrm{eV}$ при неизменном объеме КТ.

Для понимания природы обнаруженного явления мы провели дополнительные расчеты, в которых учитывалась только гидростатическая компонента тензора деформаций. Результаты таких расчетов показаны на рис. 2 светлыми символами. Видно, что в этом приближении ни энергия электронов, ни энергия дырок, ни соответственно энергия экситонов не имеют минимума, т.е. наличие оптимального формфактора пирамидальной КТ вызвано сложной структурой напряженного состояния.

Очевидным и естественным подходом к изменению локализации носителей заряда в КТ является изменение ее размеров. При этом усиление локализации достигается исключительно за счет уменьшения энергии размерного квантования. Этот подход, однако, имеет два существенных ограничения. Во-первых, заданная и, возможно, неоптимальная структура поля упругих деформаций при изменении объема КТ существенно не изменяется. Во-вторых, увеличение размеров КТ выше определенного предела неизбежно ведет к формированию дислокаций несоответствия [16,17], что обычно неприемлемо для использования КТ в электронных и оптоэлектронных приборах. Важным достоинством усиления локализации носителей заряда в КТ за счет эффекта формы является то, что этот процесс не должен приводить к формированию дислокаций несоответствия, поскольку полная механическая энергия системы при этом процессе уменьшается.

Результаты расчета энергии упругой деформации для различных соотношений высоты типичных пирамидальных КТ InAs показали, что при постоянном объеме КТ можно добиться более глубокой локализации носителей за счет реализации оптимального отношения высоты пирамиды к ее основанию $H / L=0.2$. Для таких КТ выигрыш в энергии может достигать $0.1 \mathrm{eV}$. Обнаруженный эффект формы не является специфическим для выбранной системы материалов. Можно с уверенностью утверждать, что сходные эффекты должны наблюдаться в КТ на основе различных материалов, включая традиционные III-V, III-N, II-VI, Si-Ge.

\section{Благодарности}

Авторы благодарны Н.А. Черкашину и А.В. Коновалову за полезные дискуссии. Авторы также выражают благодарность Н.А. Берту, В.Н. Неведомскому, 
А.Л. Колесниковой, А.Е. Романову, В.В. Преображенскому, Б.Р. Семягину и М.А. Путято за многолетнее сотрудничество в исследовании квантовых точек.

\section{Финансирование работы}

Работа выполнена при поддержке Российского фонда фундаментальных исследований (грант 19-32-90116).

\section{Конфликт интересов}

Авторы заявляют, что у них нет конфликта интересов.

\section{Список литературы}

[1] Ж.И. Алферов, ФТП, 32 (1), 3 (1998). [Zh.I. Alferov, Semiconductors, 32 (1), 1 (1998). DOI: 10.1134/1.1187350].

[2] M. Bayer, Ann. Phys. (Berlin), 531, 1900039 (2019). DOI: 10.1002/andp.201900039

[3] I.N. Stranski, L. Krastanow, Abhandlungen der Mathematisch-Naturwissenschaftlichen Klasse IIb. Akad. Wiss. (Wien), 146, 797 (1938).

[4] S. Adachi, Physical properties of III-V semiconductor compounds: InP, InAs, GaAs, GaP, InGaAs, and InGaAsP (John Wiley \& Sons, N.Y., 1992).

[5] K.E. Sautter, K.D. Vallejo, P.J. Simmonds, J. Appl. Phys., 128, 031101 (2020). DOI: 10.1063/5.0012066

[6] S. Ruvimov, P. Werner, K. Scheerschmidt, U. Gosele, J. Heydenreich, U. Richter, N.N. Ledentsov, M. Grundmann, D. Bimberg, V.M. Ustinov, A.Yu. Egorov, P.S. Kop'ev, Zh.I. Alferov, Phys. Rev. B, 51, 14766 (1995). DOI: 10.1103/PhysRevB.51.14766

[7] В.Н. Неведомский, Н.А. Берт, В.В. Чалдышев, В.В. Преображенский, М.А. Путято, Б.Р. Семягин, ФТП, 43 (12), 1662 (2009). [V.N. Nevedomskii, N.A. Bert, V.V. Chaldyshev, V.V. Preobrazhenskii, M.A. Putyato, B.R. Semyagin, Semiconductors, 43 (12), 1617 (2009). DOI: $10.1134 / \mathrm{S} 1063782609120082]$.

[8] N. Cherkashin, S. Reboh, M.J. Hÿtch, A. Claverie, V.V. Preobrazhenskii, M.A. Putyato, B.R. Semyagin, V.V. Chaldyshev, Appl. Phys. Lett., 102, 173115 (2013). DOI: $10.1063 / 1.4804380$

[9] I. Daruka, J. Tersoff, A.-L. Barabási, Phys. Rev. Lett., 82, 2753 (1999). DOI: 10.1103/PhysRevLett.82.2753

[10] P. Kratzer, Q.K.K. Liu, P. Acosta-Diaz, C. Manzano, G. Costantini, R. Songmuang, A. Rastelli, O.G. Schmidt, K. Kern, Phys. Rev. B, 73, 205347 (2006). DOI: 10.1103/PhysRevB.73.205347

[11] A. Kosarev, V.V. Chaldyshev, Appl. Phys. Lett., 117, 202103 (2020). DOI: $10.1063 / 5.0032110$

[12] O. Stier, M. Grundmann, D. Bimberg, Phys. Rev. B, 59, 5688 (1999). DOI: 10.1103/PhysRevB.59.5688

[13] J.D. Eshelby, Proc. R. Soc. Lond. A, 241, 376 (1957). DOI: 10.1098/rspa.1957.0133

[14] J.D. Eshelby, Proc. R. Soc. Lond. A, 252, 561 (1959). DOI: $10.1098 /$ rspa. 1959.0173

[15] Н.А. Берт, А.Л. Колесникова, А.Е. Романов, В.В. Чалдышев, ФТТ, 44 (12), 2139 (2002). [N.A. Bert, A.L. Kolesnikova, A.E. Romanov, V.V. Chaldyshev, Phys. Solid State, 44 (12), 2240 (2002). DOI: 10.1134/1.1529918].
[16] Н.А. Берт, А.Л. Колесникова, В.Н. Неведомский, В.В. Преображенский, М.А. Путято, А.Е. Романов, В.М. Селезнев, Б.Р. Семягин, В.В. Чалдышев, ФТП, 43 (10), 1426 (2009). [N.A. Bert, A.L. Kolesnikova, V.N. Nevedomsky, V.V. Preobrazhenskii, M.A. Putyato, A.E. Romanov, V.M. Seleznev, B.R. Semyagin, V.V. Chaldyshev, Semiconductors, 43 (10), 1387 (2009). DOI: $10.1134 / \mathrm{S} 1063782609100236]$.

[17] V.V. Chaldyshev, N.A. Bert, A.L. Kolesnikova, A.E. Romanov, Phys. Rev. B, 79, 233304 (2009). DOI: 10.1103/PhysRevB.79.233304 